\title{
2D ELEMENTARY CELLULAR AUTOMATA WITH FOUR NEIGHBORS
}

\author{
JOSÉ ANTÓNIO FREITAS \\ Escola Secundária Caldas de Vizela, Rua Joaquim Costa Chicória 1, \\ Caldas de Vizela, 4815-513 Vizela, Portugal \\ RICARDO SEVERINO \\ CIMA, Research Centre in Mathematics and Applications \\ Colégio Luís Verney, Rua Romão Ramalho 59 \\ Évora, 7000-671 Évora, Portugal \\ and \\ Department of Mathematics and Applications, \\ University of Minho, \\ 4710-057 Braga, Portugal
}

\begin{abstract}
This paper is concerned with the study of square boolean synchronous four-neighbor peripheral cellular automata. It is first shown that, due to conjugation and plane reflection symmetry transformations, the number of dynamically nonequivalent such automata is equal to 4856 . The cellular automata for which the homogeneous final states play a significant role are then identified. Finally, it is shown that, contrary to what happens in the case of one-dimensional boolean three-neighbor cellular automata, for some peripheral automata there is coexistence between a homogeneous final state and other dynamics.
\end{abstract}

\section{Introduction}

Despite their simple basic components, cellular automata can exhibit a variety of complex dynamical behavior. This became apparent with the pioneering work of Stephen Wolfram, who, around 1980, made extensive simulations with one-dimensional boolean three-neighbor cellular automata, usually known as elementary cellular automata (ECA). Since then, many studies of more complicated cellular automata are still concerned with fitting their time evolution into one of the typical behaviors known for the simplest 1D situation. However, it is our belief that the complexity of one-dimensional and higher dimensional automata can differ significantly, and that it is worth investigating the dynamics of $2 \mathrm{D}$ cellular automata searching for some kind of behavior not yet seen with line lattices.

The present work is concerned with the study of a special class of $2 \mathrm{D}$ automata: square boolean fourneighbor cellular automata. It is shown that, due to the plane reflection symmetry transformations, the number of dynamically nonequivalent rules for this type of automata is "sufficiently small" to enable a detailed study of all of them. In particular, we are able to identify all the cellular automata of this type for which homogeneous configurations play a significant role. Moreover, our computational experiments show that some of these cellular automata have a singular characteristic: they exhibit coexistence between a homogeneous final state and other dynamics. 


\section{Two-dimensional boolean four-neighbor cellular automata}

We consider finite $n \times m$ boolean synchronous cellular automata with a peripheral neighborhood, i.e. automata in which the state of a cell at time $t+1$ depends on the states of its four closest neighbors at the previous time $t$. If we denote by

$$
\mathrm{A}(t)=a_{i, j}(t), \quad i=1, \cdots, n, j=1, \cdots, m,
$$

the system state configuration at time $t$, then the state of the site $(i, j)$ at time $t+1, a_{i, j}(t+1)$, is a boolean function $\phi$ (the so-called local update rule) of four variables:

$$
a_{i, j}(t+1)=\phi\left(a_{i-1, j}(t), a_{i, j-1}(t), a_{i, j+1}(t), a_{i+1, j}(t)\right) .
$$

Also, we prescribe periodic boundary conditions when updating the cells at the boundaries of the rectangle.

Each configuration is, in this case, a $n \times m$ binary matrix. If we denote by $\Sigma$ the set of all such configurations, formula (2) defines the so-called global transition function $\Phi: \Sigma \rightarrow \Sigma$.

Following [Wolfram, 1984b], we can associate a code number with each cellular automaton. First, we fix the following order for the 16 different possible neighborhoods, with light gray meaning 0 and black meaning 1:

$$
\begin{array}{cccc}
\text { neighborhood }_{0}=\square & \text { neighborhood }_{1}=\square & \text { neighborhood }_{2}=\square & \text { neighborhood }_{3}=\square \\
\text { neighborhood }_{4}=\square & \text { neighborhood }_{5}=\square & \text { neighborhood }_{6}=\square & \text { neighborhood }_{7}=\square \\
\text { neighborhood }_{8}=\square & \text { neighborhood }_{9}=\square & \text { neighborhood }_{10}=\square & \text { neighborhood }_{11}=\square \\
\text { neighborhood }_{12}=\square & \text { neighborhood }_{13}=\square & \text { neighborhood }_{14}=\square & \text { neighborhood }_{15}=\square
\end{array}
$$

With this ordering of the different possible neighborhoods, we then associate, to each boolean function $\phi$, the integer number $N(\phi)$ given by:

$$
N(\phi)=\sum_{k=0}^{15} \phi\left(\text { neighborhood }_{k}\right) 2^{k} .
$$

In what follows, we will indistinctly refer to a cellular automaton by the associated boolean function $\phi$, the global function $\Phi$, or the integer code $N(\phi)$.

\section{Dynamically equivalent cellular automata}

The characterization of the time evolution of a cellular automaton must be independent of the chosen color scheme and point of view; hence, one can introduce some basic transformations between configurations and declare as dynamically equivalent those cellular automata that preserve these transformations. In the case of one-dimensional ECA, these transformations can be a conjugacy, a left-right reflection or the composition of both. The use of these transformations allows us to consider only 88 dynamically nonequivalent rules, instead of the total number of 256 different rules; see [Walker \& Aadryan, 1971], [Li \& Packard, 1990], [Wuensche \& Lesser, 1992], [Chua et al., 2004, 2005], [Chua et al., 2007], and [Guan et al., 2007]. In the plane case we are studying here, there are other transformations to be taken into account: besides the conjugacy and the left-right reflection, we also have an up-down reflection and, for square lattices, a diagonal reflection may also be added. Naturally, we also have to consider all the possible compositions of any of these transformations.

In what follows, we restrict our study to square $n \times n$ cellular automata.

Definition 3.1. We say that two configurations $\mathrm{A}$ and $\mathrm{A}^{\prime}$ are conjugate, and write $\mathrm{A} \sim_{\mathrm{c}} \mathrm{A}^{\prime}$, if $a_{i, j}^{\prime}=\bar{a}_{i, j}$, for $i, j=1, \ldots, n$, with $\overline{0}=1$ and $\overline{1}=0$ the usual conjugacy boolean operation. 
Next, we introduce the basic plane symmetry transformations.

Definition 3.2. Given two configurations $A$ and $A^{\prime}$ :

- we say that they are left-right symmetric, and write $\mathrm{A} \sim_{\operatorname{lr}} \mathrm{A}^{\prime}$, if $a_{i, j}^{\prime}=a_{n+1-i, j}$, for $i, j=1, \ldots, n$;

- we say that they are up-down symmetric, and write $\mathrm{A} \sim_{\text {ud }} \mathrm{A}^{\prime}$, if $a_{i, j}^{\prime}=a_{i, n+1-j}$, for $i, j=1, \ldots, n$;

- we say that they are diagonal symmetric, and write $\mathrm{A} \sim_{\mathrm{d}} \mathrm{A}^{\prime}$, if $a_{i, j}^{\prime}=a_{j, i}$, for $i, j=1, \ldots, n$.

It should be noted that there is no need to consider the anti-diagonal symmetry transformation since it can be obtained as a composition of the other three.

Definition 3.3. Given two cellular automata, $\phi$ and $\phi^{\prime}$, we say that they are conjugate equivalent, and write $\phi \sim_{c} \phi^{\prime}$, if, for any two conjugate configurations $A \sim_{c} A^{\prime}$, we have $\Phi(A) \sim_{c} \Phi^{\prime}\left(A^{\prime}\right)$.

Definition 3.4. Given two cellular automata, $\phi$ and $\phi^{\prime}$ :

- we say that they are left-right equivalent, and write $\phi \sim_{\operatorname{lr}} \phi^{\prime}$ if, given any two left-right symmetric configurations $A \sim_{\operatorname{lr}} A^{\prime}$, we have $\Phi(A) \sim_{\operatorname{lr}} \Phi^{\prime}\left(A^{\prime}\right)$;

- we say that they are up-down equivalent, and write $\phi \sim_{\text {ud }} \phi^{\prime}$ if, given any two up-down symmetric configurations $\mathrm{A} \sim_{\text {ud }} \mathrm{A}^{\prime}$, we have $\Phi(\mathrm{A}) \sim_{\text {ud }} \Phi^{\prime}\left(\mathrm{A}^{\prime}\right)$;

- we say that they are diagonal equivalent, and write $\phi \sim_{d} \phi^{\prime}$ if, given any two diagonal symmetric configurations $A \sim_{d} A^{\prime}$, we have $\Phi(A) \sim_{d} \Phi^{\prime}\left(A^{\prime}\right)$.

In what follows, given two cellular automata, $\phi$ and $\phi^{\prime}$, we consider the binary representation of their integer codes, $N(\phi)=\left(b_{15} \ldots b_{0}\right)_{2}$ and $N\left(\phi^{\prime}\right)=\left(b_{15}^{\prime} \ldots b_{0}^{\prime}\right)_{2}$. The following four propositions characterize the above basic equivalences of cellular automata in terms of the binary representation of their integer codes. Since the proofs of the propositions are all very similar, we will only present in detail the proof of the last one.

Proposition 1. Two cellular automata, $\phi$ and $\phi^{\prime}$, are conjugate equivalent, $\phi \sim_{\mathrm{c}} \phi^{\prime}$, if and only if the digits $b_{i}$ and $b_{i}^{\prime}$ in the binary representation of their integer codes satisfy

$$
\begin{array}{llll}
b_{0}^{\prime}=\bar{b}_{15} & b_{1}^{\prime}=\bar{b}_{14} & b_{2}^{\prime}=\bar{b}_{13} & b_{3}^{\prime}=\bar{b}_{12} \\
b_{4}^{\prime}=\bar{b}_{11} & b_{5}^{\prime}=\bar{b}_{10} & b_{6}^{\prime}=\bar{b}_{9} & b_{7}^{\prime}=\bar{b}_{8} \\
b_{8}^{\prime}=\bar{b}_{7} & b_{9}^{\prime}=\bar{b}_{6} & b_{10}^{\prime}=\bar{b}_{5} & b_{11}^{\prime}=\bar{b}_{4} \\
b_{12}^{\prime}=\bar{b}_{3} & b_{13}^{\prime}=\bar{b}_{2} & b_{14}^{\prime}=\bar{b}_{1} & b_{15}^{\prime}=\bar{b}_{0}
\end{array}
$$

Proposition 2. Two cellular automata, $\phi$ and $\phi^{\prime}$, are left-right equivalent, $\phi \sim_{\operatorname{lr}} \phi^{\prime}$, if and only if the digits $b_{i}$ and $b_{i}^{\prime}$ in the binary representation of their integer codes satisfy

$$
\begin{array}{llll}
b_{0}^{\prime}=b_{0} & b_{1}^{\prime}=b_{1} & b_{2}^{\prime}=b_{4} & b_{3}^{\prime}=b_{5} \\
b_{4}^{\prime}=b_{2} & b_{5}^{\prime}=b_{3} & b_{6}^{\prime}=b_{6} & b_{7}^{\prime}=b_{7} \\
b_{8}^{\prime}=b_{8} & b_{9}^{\prime}=b_{9} & b_{10}^{\prime}=b_{12} & b_{11}^{\prime}=b_{13} \\
b_{12}^{\prime}=b_{10} & b_{13}^{\prime}=b_{11} & b_{14}^{\prime}=b_{14} & b_{15}^{\prime}=b_{15}
\end{array}
$$

Proposition 3. Two cellular automata, $\phi$ and $\phi^{\prime}$, are up-down equivalent, $\phi \sim_{\mathrm{ud}} \phi^{\prime}$, if and only if the digits $b_{i}$ and $b_{i}^{\prime}$ in the binary representation of their integer codes satisfy

$$
\begin{array}{llll}
b_{0}^{\prime}=b_{0} & b_{1}^{\prime}=b_{8} & b_{2}^{\prime}=b_{2} & b_{3}^{\prime}=b_{10} \\
b_{4}^{\prime}=b_{4} & b_{5}^{\prime}=b_{12} & b_{6}^{\prime}=b_{6} & b_{7}^{\prime}=b_{14} \\
b_{8}^{\prime}=b_{1} & b_{9}^{\prime}=b_{9} & b_{10}^{\prime}=b_{3} & b_{11}^{\prime}=b_{11} \\
b_{12}^{\prime}=b_{5} & b_{13}^{\prime}=b_{13} & b_{14}^{\prime}=b_{7} & b_{15}^{\prime}=b_{15}
\end{array}
$$


Proposition 4. Two cellular automata, $\phi$ and $\phi^{\prime}$, are diagonal equivalent, $\phi \sim_{\mathrm{d}} \phi^{\prime}$, if and only if the digits $b_{i}$ and $b_{i}^{\prime}$ in the binary representation of their integer codes satisfy

$$
\begin{array}{llll}
b_{0}^{\prime}=b_{0} & b_{1}^{\prime}=b_{2} & b_{2}^{\prime}=b_{1} & b_{3}^{\prime}=b_{3} \\
b_{4}^{\prime}=b_{8} & b_{5}^{\prime}=b_{10} & b_{6}^{\prime}=b_{9} & b_{7}^{\prime}=b_{11} \\
b_{8}^{\prime}=b_{4} & b_{9}^{\prime}=b_{6} & b_{10}^{\prime}=b_{5} & b_{11}^{\prime}=b_{7} \\
b_{12}^{\prime}=b_{12} & b_{13}^{\prime}=b_{14} & b_{14}^{\prime}=b_{13} & b_{15}^{\prime}=b_{15}
\end{array}
$$

Proof. Let $\mathrm{A}=\left(a_{i, j}\right)$ and $\mathrm{A}^{\prime}=\left(a_{i, j}^{\prime}\right)$ be two diagonally equivalent configurations, i.e., $a_{i, j}^{\prime}=a_{j, i}$, and consider their images by the automata $\Phi$ and $\Phi^{\prime}$, respectively, $\tilde{\mathrm{A}}=\Phi(\mathrm{A})=\left(\tilde{a}_{i, j}\right)$ and $\tilde{\mathrm{A}}^{\prime}=\Phi^{\prime}\left(\mathrm{A}^{\prime}\right)=\left(\tilde{a}_{i, j}^{\prime}\right)$.

If the automata are diagonally equivalent, then $\tilde{\mathrm{A}} \sim_{d} \tilde{\mathrm{A}}^{\prime}$, i.e. we must have $\tilde{a}_{i, j}^{\prime}=\tilde{a}_{j, i}$. But,

$$
\begin{aligned}
\tilde{a}_{i, j}^{\prime}=\tilde{a}_{j, i} & \Leftrightarrow \phi^{\prime}\left(a_{i-1, j}^{\prime}, a_{i, j-1}^{\prime}, a_{i, j+1}^{\prime}, a_{i+1, j}^{\prime}\right)=\phi\left(a_{j-1, i}, a_{j, i-1}, a_{j, i+1}, a_{j+1, i}\right) \\
& \Leftrightarrow \phi^{\prime}\left(a_{i-1, j}^{\prime}, a_{i, j-1}^{\prime}, a_{i, j+1}^{\prime}, a_{i+1, j}^{\prime}\right)=\phi\left(a_{i, j-1}^{\prime}, a_{i-1, j}^{\prime}, a_{i+1, j}^{\prime}, a_{i, j+1}^{\prime}\right) .
\end{aligned}
$$

Hence, if the automata $\phi$ and $\phi^{\prime}$ are diagonally equivalent, we must have

$$
\phi^{\prime}(x, y, z, w)=\phi(y, x, w, z), \quad x, y, z, w \in\{0,1\} .
$$

From (4), it follows that:

$$
\begin{array}{ll}
b_{0}^{\prime}=\phi^{\prime}(0,0,0,0)=\phi(0,0,0,0)=b_{0} & b_{1}^{\prime}=\phi^{\prime}(0,0,0,1)=\phi(0,0,1,0)=b_{2} \\
b_{2}^{\prime}=\phi^{\prime}(0,0,1,0)=\phi(0,0,0,1)=b_{1} & b_{3}^{\prime}=\phi^{\prime}(0,0,1,1)=\phi(0,0,1,1)=b_{3} \\
b_{4}^{\prime}=\phi^{\prime}(0,1,0,0)=\phi(1,0,0,0)=b_{8} & b_{5}^{\prime}=\phi^{\prime}(0,1,0,1)=\phi(1,0,1,0)=b_{10} \\
b_{6}^{\prime}=\phi^{\prime}(0,1,1,0)=\phi(1,0,0,1)=b_{9} & b_{7}^{\prime}=\phi^{\prime}(0,1,1,1)=\phi(1,0,1,1)=b_{11} \\
b_{8}^{\prime}=\phi^{\prime}(1,0,0,0)=\phi(0,1,0,0)=b_{4} & b_{9}^{\prime}=\phi^{\prime}(1,0,0,1)=\phi(0,1,1,0)=b_{6} \\
b_{10}^{\prime}=\phi^{\prime}(1,0,1,0)=\phi(0,1,0,1)=b_{5} & b_{11}^{\prime}=\phi^{\prime}(1,0,1,1)=\phi(0,1,1,1)=b_{7} \\
b_{12}^{\prime}=\phi^{\prime}(1,1,0,0)=\phi(1,1,0,0)=b_{12} & b_{13}^{\prime}=\phi^{\prime}(1,1,0,1)=\phi(1,1,1,0)=b_{14} \\
b_{14}^{\prime}=\phi^{\prime}(1,1,1,0)=\phi(1,1,0,1)=b_{13} & b_{15}^{\prime}=\phi^{\prime}(1,1,1,1)=\phi(1,1,1,1)=b_{15} .
\end{array}
$$

\begin{tabular}{|c|c|c|c|c|}
\hline c & Ir & ud & $d$ & \\
\hline$c \cdot I r$ & $c \cdot u d$ & $c \cdot d$ & $\mathrm{Ir} \cdot \mathrm{ud}$ & $\mathrm{ud} \cdot \mathrm{d}$ \\
\hline $\begin{array}{l}c \cdot \operatorname{lr} \cdot \text { ud } \\
c \cdot \operatorname{lr} \cdot \mathrm{ud} \cdot \mathrm{d}\end{array}$ & $c \cdot \operatorname{lr} \cdot d$ & $c \cdot u d \cdot d$ & $\mathrm{Ir} \cdot \mathrm{ud} \cdot \mathrm{d}$ & \\
\hline
\end{tabular}

Conversely, if the digits $b_{i}$ and $b_{i}^{\prime}$ satisfy the relations (5), then relation (4) holds and this, in turn, is all we need to conclude that $\tilde{a}_{i, j}^{\prime}=\tilde{a}_{j, i}$, i.e. that the automata are equivalent.

Definition 3.5. Given two cellular automata, $\phi$ and $\phi^{\prime}$, we say that they are dynamically equivalent if, given any two configurations $A$ and $A^{\prime}$ such that $A^{\prime}$ is obtained from $A$ by a successive application of any number of the four basic transformations then, $\Phi(\mathrm{A})$ and $\Phi^{\prime}\left(\mathrm{A}^{\prime}\right)$ are related by exactly the same transformations.

The following result is important because it identifies, which, among all different compositions of the four referred basic transformations, are different. For simplicity, we introduce the notation $x \cdot y$ to denote the successive application of any basic transformations $\sim_{\mathrm{x}}, \sim_{\mathrm{y}}$.

Proposition 5. There are 15 different dynamical equivalences between cellular automata, which can be written as follows: 
Proof. By using Propositions 1-4, one can easily verify that each of the basic transformations is its own inverse:

$$
c \cdot c=i d \quad \text { Ir } \cdot I r=i d \quad u d \cdot u d=i d \quad d \cdot d=i d,
$$

where id denotes the identity transformation, and also that the following identities hold:

$$
\begin{aligned}
& \mathrm{Ir} \cdot \mathrm{c}=\mathrm{c} \cdot \mathrm{Ir} \quad \mathrm{ud} \cdot \mathrm{c}=\mathrm{c} \cdot \mathrm{ud} \quad \mathrm{d} \cdot \mathrm{c}=\mathrm{c} \cdot \mathrm{d} \quad \mathrm{ud} \cdot \mathrm{Ir}=\mathrm{Ir} \cdot \mathrm{ud} \\
& d \cdot \operatorname{Ir}=u d \cdot d \quad d \cdot u d=I r \cdot d
\end{aligned}
$$

It follows from (8) that any composition $x_{1} \cdot x_{2} \cdot \ldots \cdot x_{p}$ with $x_{i} \in\{c, \mid r, u d, d\}$ can be rearranged in the form

$$
\underbrace{c \cdot \ldots \cdot c}_{p_{1}} \cdot \underbrace{\operatorname{lr} \cdot \ldots \cdot \operatorname{lr}}_{p_{2}} \cdot \underbrace{\mathrm{ud} \cdot \ldots \cdot \mathrm{ud}}_{p_{3}} \cdot \underbrace{\mathrm{d} \cdot \ldots \cdot \mathrm{d}}_{p_{4}}
$$

with $p_{i} \geq 0$ and $p_{1}+p_{2}+p_{3}+p_{4}=p$. With the use of (7), it becomes clear that $\mathrm{x}_{1} \cdot \mathrm{x}_{2} \cdot \ldots \cdot \mathrm{x}_{p}$ is equal to one of the transformations listed in (6). Finally, it is a trivial exercise to show that these transformations are, indeed, different; see, e.g. the example below.

Example 3.1. Consider the cellular automaton $\phi$ with integer code $N(\phi)=123$. From the previous results, we know that there are at most 15 cellular automata dynamically equivalent to $\phi$. Their codes are:

$\begin{array}{lll}123 \sim_{\text {c }} 8703 & 123 \sim_{\text {Ir }} 111 & 123 \sim_{\text {ud }} 5457 \\ 123 \sim_{\text {d }} 1805 & 123 \sim_{\text {c.lr }} 2559 & 123 \sim_{\text {c.ud }} 30039 \\ 123 \sim_{\text {c.d }} 20255 & 123 \sim_{\text {Ir.ud }} 5445 & 123 \sim_{\text {Ir.d }} 1551 \\ 123 \sim_{\text {ud.d }} 4913 & 123 \sim_{\text {c.lr.ud }} 23895 & 123 \sim_{\text {c.lr.d }} 3999 \\ 123 \sim_{\text {c.ud.d }} 29495 & 123 \sim_{\text {Ir.ud.d }} 4659 & 123 \sim_{\text {c.lr.ud.d }} 13239\end{array}$

In Appendix A, we list all the dynamically nonequivalent cellular automata rules, obtained by applying the 15 equivalence transformations referred to in Proposition 5 to the 65536 different automata. As a result of these computations, we can state the following result:

Theorem 1. There are 4856 dynamically nonequivalent square boolean synchronous peripheral cellular automata.

We claim that the number 4856 is sufficiently small to allow a detailed study of the dynamics of these automata, in a manner similar to what was done for the case of ECA. Moreover, this is almost surely the only family of two-dimensional cellular automata that we may still be able to investigate explicitly. Note that, according to Proposition 5, the number of nonequivalent 2D five-neighbor boolean cellular automata is, already, at least 286331153.

Next, we will identify the cellular automata for which a homogeneous configuration is dynamically relevant.

\section{Class-1 homogeneous cellular automata}

In 1984, Wolfram [Wolfram, 1984a] proposed a classification of one-dimensional boolean three-neighbor cellular automata into four different classes, based on the analysis of the behavior of patterns generated by their time evolution. Although this classification was given for a particular type of system, it became widely accepted also for more general cellular automata. The first class identified by Wolfram corresponds to the following behavior: starting from typical initial configurations, the cellular automaton evolves to homogeneous final states [Packard \& Wolfram, 1985], where these final states can be either a fixed point, a pair of fixed points, or a 2-cycle. Since, for any automaton, one of the three situations cited above is always an attractor, the key point here is the starting from typical initial conditions: class-1 cellular automata are those rules for which the relative size of the set of configurations leading to the homogeneous final state, 


\section{JOSÉ ANTÓNIO FREITAS and RICARDO SEVERINO}

i.e. the relative size of the basin of attraction of the homogeneous final state, tends to 1 as the number of sites of the system increases. Our computational experiments indicate that of the 2D square boolean synchronous peripheral cellular automata with periodic boundary conditions studied in this paper, 353 correspond to class-1 dynamics.

First, we list the cellular automata codes corresponding to a fixed point homogeneous final state:

Table 1. Class-1 cellular automata codes with fixed point homogeneous final state.

\begin{tabular}{cccccccccccccc}
\hline 0 & 8 & 40 & 64 & 72 & 104 & 128 & 136 & 168 & 192 & 200 & 232 & 552 & 576 \\
584 & 616 & 640 & 648 & 680 & 704 & 712 & 744 & 1056 & 1064 & 1120 & 1128 & 1152 & 1160 \\
1184 & 1192 & 1216 & 1224 & 1248 & 1256 & 1632 & 1640 & 1664 & 1672 & 1696 & 1704 & 1728 & 1736 \\
1760 & 1768 & 2176 & 2184 & 2208 & 2216 & 2240 & 2248 & 2272 & 2280 & 2720 & 2728 & 2752 & 2760 \\
2784 & 2792 & 3232 & 3240 & 3296 & 3304 & 3808 & 3816 & 5248 & 5256 & 5312 & 5320 & 5760 & 5768 \\
5824 & 5832 & 6272 & 6280 & 6304 & 6312 & 6336 & 6344 & 6368 & 6376 & 6816 & 6824 & 6848 & 6856 \\
6880 & 6888 & 7328 & 7392 & 7904 & 10240 & 10248 & 10280 & 10304 & 10312 & 10344 & 10368 & 10376 & 10408 \\
10432 & 10440 & 10472 & 10752 & 10760 & 10792 & 10816 & 10824 & 10856 & 10880 & 10888 & 10920 & 10944 & 10952 \\
10984 & 11272 & 11296 & 11304 & 11336 & 11360 & 11368 & 11392 & 11400 & 11424 & 11432 & 11456 & 11464 & 11488 \\
11496 & 11784 & 11808 & 11816 & 11848 & 11872 & 11880 & 11904 & 11912 & 11936 & 11944 & 11968 & 11976 & 12000 \\
15488 & 15496 & 15552 & 15560 & 16000 & 16008 & 16064 & 26752 & 26760 & 26792 & 26816 & 26824 & 26856 & 27304 \\
27328 & 27336 & 27368 & 27808 & 27816 & 27872 & 27880 & 28384 & & & & & & \\
\hline
\end{tabular}

Next, we list the cellular automata codes for which there is coexistence of two fixed points as homogeneous final states:

Table 2. Class-1 cellular automata codes with coexistence of two fixed points as homogeneous final states.

\begin{tabular}{llllllllllllll}
\hline 32768 & 32776 & 32808 & 32832 & 32840 & 32872 & 32896 & 32904 & 32936 & 32960 & 32968 & 33000 & 33320 & 33344 \\
33352 & 33384 & 33408 & 33416 & 33448 & 33472 & 33480 & 33512 & 33824 & 33832 & 33888 & 33896 & 33920 & 33928 \\
33952 & 33960 & 33984 & 33992 & 34016 & 34024 & 34400 & 34408 & 34432 & 34440 & 34464 & 34472 & 34496 & 34504 \\
34528 & 34536 & 34944 & 34952 & 34976 & 34984 & 35008 & 35016 & 35040 & 35048 & 35488 & 35496 & 35520 & 35528 \\
35552 & 35560 & 36000 & 36008 & 36064 & 36072 & 36576 & 38016 & 38024 & 38080 & 38088 & 38528 & 38536 & 38592 \\
38600 & 39040 & 39048 & 39072 & 39080 & 39104 & 39112 & 39136 & 39144 & 39584 & 39592 & 39616 & 39624 & 39648 \\
40096 & 40160 & 40672 & 43008 & 43016 & 43048 & 43072 & 43080 & 43112 & 43136 & 43144 & 43176 & 43200 & 43208 \\
43520 & 43528 & 43560 & 43584 & 43592 & 43624 & 43648 & 43656 & 43688 & 43712 & 43720 & 44040 & 44064 & 44072 \\
44104 & 44128 & 44136 & 44160 & 44168 & 44192 & 44200 & 44224 & 44232 & 44256 & 44552 & 44576 & 44584 & 44616 \\
44640 & 44672 & 44680 & 44704 & 44712 & 44736 & 44744 & 44768 & 48256 & 48264 & 48320 & 48328 & 48768 & 48776 \\
48832 & 59520 & 59528 & 59560 & 59584 & 59592 & 60096 & 60104 & 60576 & 60640 & & & & \\
\hline
\end{tabular}

Finally, we list the cellular automata codes corresponding to a 2-cycle homogeneous final state: 
Table 3. Class-1 cellular automata codes with 2-cycle homogeneous final state.

\begin{tabular}{cccccccccccccccc}
\hline 3 & 7 & 23 & 25 & 27 & 31 & 63 & 67 & 69 & 71 & 87 & 89 & 91 & 95 & 127 & 287 \\
297 & 303 & 317 & 319 & 327 & 329 & 333 & 335 & 351 & 367 & 415 & 447 & 479 & 579 & 583 & 599 \\
603 & 607 & 815 & 829 & 863 & 879 & 991 & 927 & 1063 & 1127 & 1339 & 1403 & 1639 & & & \\
\hline
\end{tabular}

It should be mentioned that, although belonging to the same class, some of these automata show a linear growing process of their basin of attraction of the homogeneous final state, in contrast with the exponential growth behavior displayed by all the elementary cellular automata.

We now describe the computational procedure used to obtain all the results that follow. Given an automaton, we denote by $B_{h}$ the basin of attraction of its homogeneous final state and by $\% B_{h}$ the relative size of $B_{h}$. To obtain a first approximation, $M_{h}$, to the length of $B_{h}$, we used 2000 random initial configurations and computed the maximum of the transient times of all of those configurations that led to the homogeneous final state; in this computation, we allowed the system to evolve for a time much larger than $M_{h}$; then, using 12000 random initial configurations, we estimated $\% B_{h}$ from the number of initial configurations that, for a time $\triangle t=1.2 M_{h}$, reached the homogeneous final state.

Example 4.1. We considered the cellular automaton $N(\phi)=44648$ and computed $\% B_{h}$ as indicated above. The results are displayed in the next figure, which clearly shows an extremely slow linear growth of $\% B_{h}$ with the size of the automaton, specially for even values of $d$.

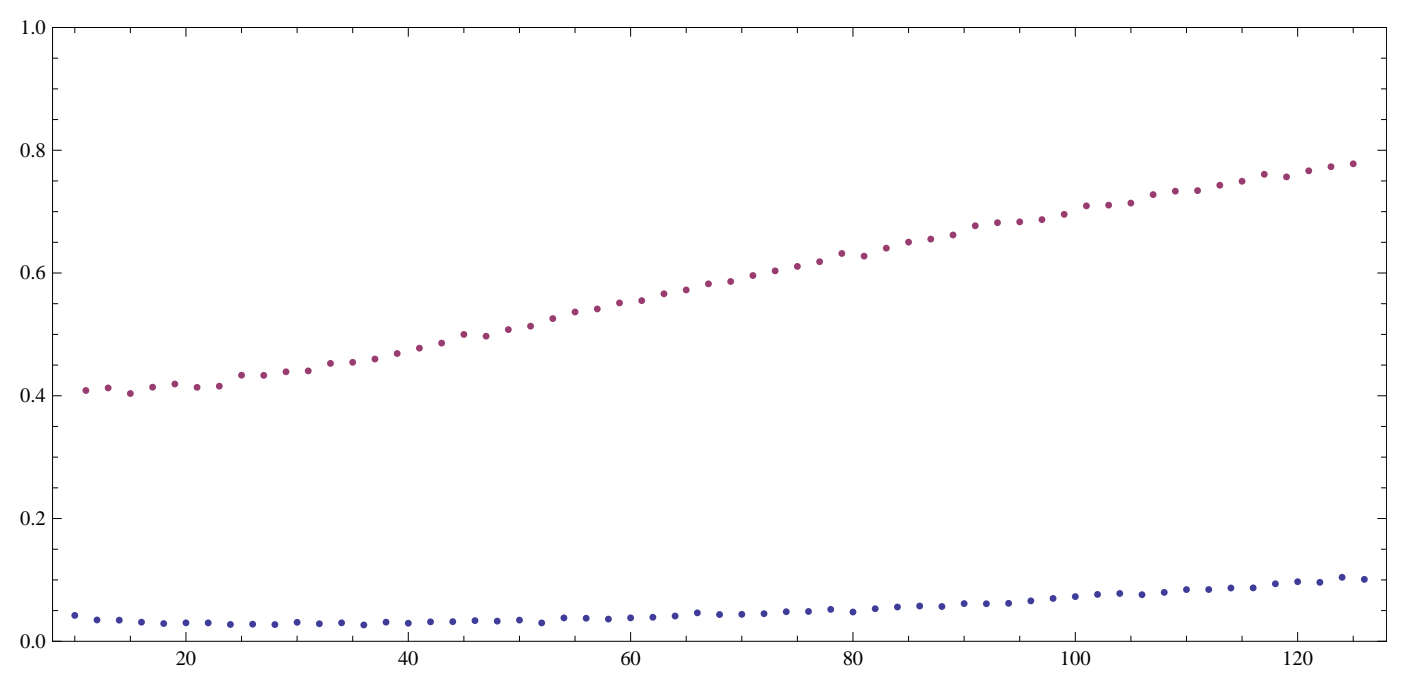

Fig. 1. Change with $d$ of the relative size of the basin of attraction of the homogeneous null final state for the $d \times d$ cellular automaton $N(\phi)=44648$.

\section{Coexistence of dynamics}

Although relevant for any computational simulation, the linear growth of the importance of the homogeneous final state with the size of the system still satisfies the original idea that defined automata of class-1. Yet, we found cellular automata for which coexistence between a homogeneous final state and other dynamics is intrinsic to the system, in the sense that, no matter how large we choose the number of their elements to be, there is always coexistence of dynamics. 
Example 5.1. We considered the cellular automaton $N(\phi)=383$ and obtained the results shown in the following figure, where we also plotted the linear fits, $L_{\text {odd }}$ and $L_{\text {even }}$, of the points corresponding to odd and even values of $d$, respectively.

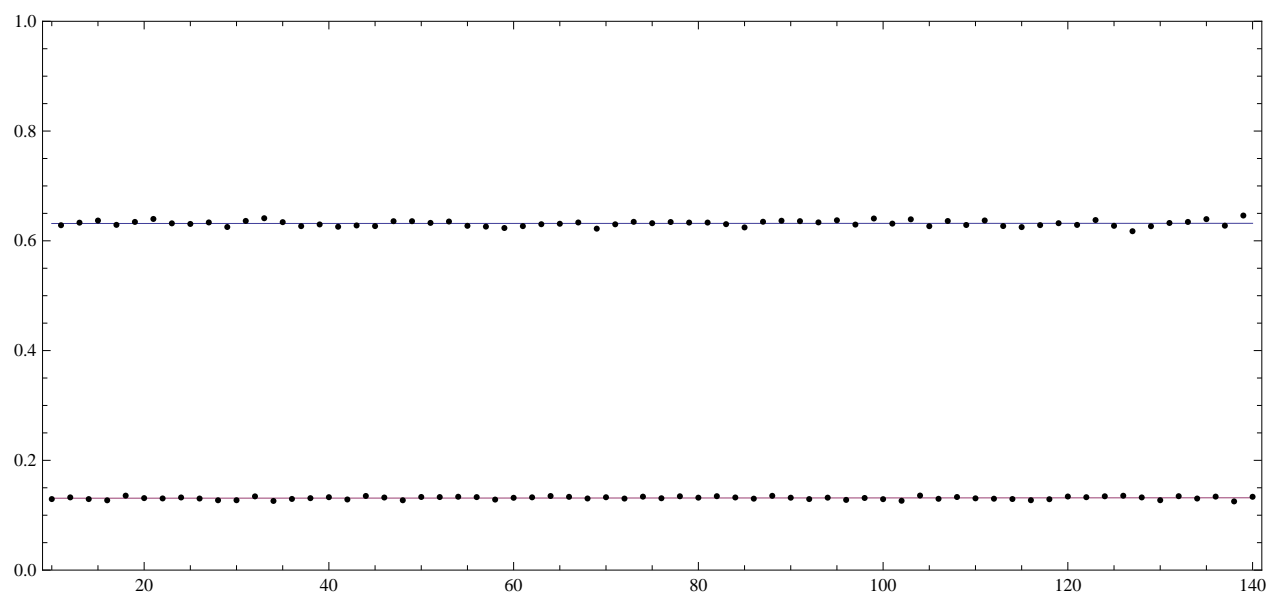

Fig. 2. Change with $d$ of the relative size of the basin of attraction of the homogeneous final state for the $d \times d$ cellular automaton $N(\phi)=383$.

The expressions found for the linear fits are given by $L_{\text {odd }}(d)=0.631536+2.45484 \times 10^{-6} d$ and $L_{\text {even }}(d)=$ $0.130931+5.87447 \times 10^{-6} d$. The very small values of both slopes allow us to say that, for each case, $d$ even and $d$ odd, the relative size of the basin of attraction of the homogeneous final state $\% B_{h}$ does not depend on $d$.

Other computational experiments led us to conclude that there exist six square boolean peripheral cellular automata with periodic boundary conditions for which the relative size of the basin of attraction of the homogeneous final state remains constant. Their codes are given in the following table.

Table 4. The square peripheral cellular automata that exhibit a homogeneous final state coexisting with other dynamics.

\begin{tabular}{llllll}
\hline 383 & 575 & 831 & 43240 & 59624 & 60072 \\
\hline
\end{tabular}

It is worth noticing that, of the listed six rules showing coexistence of dynamics, the first three have a 2-cycle as homogeneous final state, while the other three have a pair of fixed points as homogeneous final states.

\section{Conclusions}

The possibility to do a detailed analysis of a family of cellular automata, as Wolfram did for the ECA, gives us a global perception of the diversity of its dynamics. However, for more complicated systems than the ones considered by Wolfram, the attempt to systematically scrutinize all the dynamics has obvious computational difficulties, due to the exponential growth of the number of elements of the family. We have shown that, due to plane symmetry transformations, the family of $2 \mathrm{D}$ square boolean peripheral automata has only 4856 dynamically nonequivalent rules. Since there are a total of 65536 different rules, this implies a saving of nearly $93 \%$ of computer time. This reasonable number of rules enabled us to analyze all of them in order to identify which ones are of class- 1 . We also showed that there are systems for which the relative size of the basin of attraction of the homogeneous final state does not depend on the number of sites of the system. This is a very surprising result, not seen for the ECA case nor, as far as we are aware, referred to for other systems and gives us the conviction that this family of automata deserves further investigation. 


\section{Appendix A}

In the following table we list the 4856 dynamically nonequivalent square boolean synchronous four-neighbor peripheral cellular automata codes. As usual, we choose the cellular automaton with the smallest code as the equivalence class representative.

Table A.1. Dinamically nonequivalent square boolean peripheral cellular automata codes; cellular automata with the smallest code were chosen as the equivalence class representatives.

\begin{tabular}{|c|c|c|c|c|c|c|c|c|c|c|c|c|c|}
\hline 0 & 1 & 2 & 3 & 6 & 7 & 8 & 9 & 10 & 11 & 14 & 15 & 20 & 21 \\
\hline 22 & 23 & 24 & 25 & 26 & 27 & 28 & 29 & 30 & 31 & 40 & 41 & 42 & 43 \\
\hline 44 & 45 & 46 & 47 & 60 & 61 & 62 & 63 & 64 & 65 & 66 & 67 & 68 & 69 \\
\hline 70 & 71 & 72 & 73 & 74 & 75 & 76 & 77 & 78 & 79 & 84 & 85 & 86 & 87 \\
\hline 88 & 89 & 90 & 91 & 92 & 93 & 94 & 95 & 104 & 105 & 106 & 107 & 108 & 109 \\
\hline 110 & 111 & 124 & 125 & 126 & 127 & 128 & 129 & 130 & 131 & 132 & 133 & 134 & 135 \\
\hline 136 & 137 & 138 & 139 & 140 & 141 & 142 & 143 & 148 & 149 & 150 & 151 & 152 & 153 \\
\hline 154 & 155 & 156 & 157 & 158 & 159 & 168 & 169 & 170 & 171 & 172 & 173 & 174 & 175 \\
\hline 188 & 189 & 190 & 191 & 192 & 193 & 194 & 195 & 196 & 197 & 198 & 199 & 200 & 201 \\
\hline 202 & 203 & 204 & 205 & 206 & 207 & 212 & 213 & 214 & 215 & 216 & 217 & 218 & 219 \\
\hline 220 & 221 & 222 & 223 & 232 & 233 & 234 & 235 & 236 & 237 & 238 & 239 & 252 & 253 \\
\hline 254 & 255 & 278 & 279 & 280 & 281 & 282 & 283 & 286 & 287 & 296 & 297 & 298 & 299 \\
\hline 300 & 301 & 302 & 303 & 316 & 317 & 318 & 319 & 322 & 323 & 326 & 327 & 328 & 329 \\
\hline 330 & 331 & 332 & 333 & 334 & 335 & 342 & 343 & 344 & 345 & 346 & 347 & 348 & 349 \\
\hline 350 & 351 & 360 & 361 & 362 & 363 & 364 & 365 & 366 & 367 & 380 & 381 & 382 & 383 \\
\hline 384 & 385 & 386 & 387 & 388 & 389 & 390 & 391 & 392 & 393 & 394 & 395 & 396 & 397 \\
\hline 398 & 399 & 404 & 405 & 406 & 407 & 408 & 409 & 410 & 411 & 412 & 413 & 414 & 415 \\
\hline 424 & 425 & 426 & 427 & 428 & 429 & 430 & 431 & 444 & 445 & 446 & 447 & 448 & 449 \\
\hline 450 & 451 & 452 & 453 & 454 & 455 & 456 & 457 & 458 & 459 & 460 & 461 & 462 & 463 \\
\hline 468 & 469 & 470 & 471 & 472 & 473 & 474 & 475 & 476 & 477 & 478 & 479 & 488 & 489 \\
\hline 490 & 491 & 492 & 493 & 494 & 495 & 508 & 509 & 510 & 552 & 553 & 554 & 555 & 556 \\
\hline 557 & 558 & 559 & 572 & 573 & 574 & 575 & 576 & 577 & 578 & 579 & 582 & 583 & 584 \\
\hline 585 & 586 & 587 & 590 & 591 & 596 & 597 & 598 & 599 & 600 & 601 & 602 & 603 & 604 \\
\hline 605 & 606 & 607 & 616 & 617 & 618 & 619 & 620 & 621 & 622 & 623 & 636 & 637 & 638 \\
\hline 640 & 641 & 642 & 643 & 644 & 645 & 646 & 647 & 648 & 649 & 650 & 651 & 652 & 653 \\
\hline 654 & 655 & 660 & 661 & 662 & 663 & 664 & 665 & 666 & 667 & 668 & 669 & 670 & 671 \\
\hline 680 & 681 & 682 & 683 & 684 & 685 & 686 & 687 & 700 & 701 & 702 & 703 & 704 & 705 \\
\hline 706 & 707 & 708 & 709 & 710 & 711 & 712 & 713 & 714 & 715 & 716 & 717 & 718 & 719 \\
\hline 724 & 725 & 726 & 727 & 728 & 729 & 730 & 731 & 732 & 733 & 734 & 735 & 744 & 745 \\
\hline 746 & 747 & 748 & 749 & 750 & 751 & 764 & 765 & 766 & 808 & 809 & 810 & 811 & 812 \\
\hline 813 & 814 & 815 & 828 & 829 & 830 & 831 & 854 & 855 & 1006 & 1007 & 1020 & 1021 & 1022 \\
\hline 1056 & 1057 & 1058 & 1059 & 1062 & 1063 & 1064 & 1065 & 1066 & 1067 & 1070 & 1071 & 1074 & 1075 \\
\hline
\end{tabular}


Table A.2. Table 1A. (continued).

\begin{tabular}{|c|c|c|c|c|c|c|c|c|c|c|c|c|c|}
\hline 1076 & 1077 & 1078 & 1079 & 1080 & 1081 & 1082 & 1083 & 1084 & 1085 & 1086 & 1120 & 1121 & 1122 \\
\hline 1123 & 1124 & 1125 & 1126 & 1127 & 1128 & 1129 & 1130 & 1131 & 1132 & 1133 & 1134 & 1135 & 1138 \\
\hline 1139 & 1140 & 1141 & 1142 & 1143 & 1144 & 1145 & 1146 & 1147 & 1148 & 1149 & 1150 & 1152 & 1153 \\
\hline 1154 & 1155 & 1156 & 1157 & 1158 & 1159 & 1160 & 1161 & 1162 & 1163 & 1164 & 1165 & 1166 & 1167 \\
\hline 1168 & 1169 & 1170 & 1171 & 1172 & 1173 & 1174 & 1175 & 1176 & 1177 & 1178 & 1179 & 1180 & 1181 \\
\hline 1182 & 1183 & 1184 & 1185 & 1186 & 1187 & 1188 & 1189 & 1190 & 1191 & 1192 & 1193 & 1194 & 1195 \\
\hline 1196 & 1197 & 1198 & 1199 & 1200 & 1201 & 1202 & 1203 & 1204 & 1205 & 1206 & 1207 & 1208 & 1209 \\
\hline 1210 & 1211 & 1212 & 1213 & 1214 & 1216 & 1217 & 1218 & 1219 & 1220 & 1221 & 1222 & 1223 & 1224 \\
\hline 1225 & 1226 & 1227 & 1228 & 1229 & 1230 & 1231 & 1232 & 1233 & 1234 & 1235 & 1236 & 1237 & 1238 \\
\hline 1239 & 1240 & 1241 & 1242 & 1243 & 1244 & 1245 & 1246 & 1247 & 1248 & 1249 & 1250 & 1251 & 1252 \\
\hline 1253 & 1254 & 1255 & 1256 & 1257 & 1258 & 1259 & 1260 & 1261 & 1262 & 1263 & 1264 & 1265 & 1266 \\
\hline 1267 & 1268 & 1269 & 1270 & 1271 & 1272 & 1273 & 1274 & 1275 & 1276 & 1277 & 1278 & 1334 & 1335 \\
\hline 1336 & 1337 & 1338 & 1339 & 1342 & 1378 & 1379 & 1382 & 1383 & 1384 & 1385 & 1386 & 1387 & 1388 \\
\hline 1389 & 1390 & 1398 & 1399 & 1400 & 1401 & 1402 & 1403 & 1404 & 1405 & 1406 & 1408 & 1409 & 1410 \\
\hline 1411 & 1412 & 1413 & 1414 & 1415 & 1416 & 1417 & 1418 & 1419 & 1420 & 1421 & 1422 & 1424 & 1425 \\
\hline 1426 & 1427 & 1428 & 1429 & 1430 & 1431 & 1432 & 1433 & 1434 & 1435 & 1436 & 1437 & 1438 & 1440 \\
\hline 1441 & 1442 & 1443 & 1444 & 1445 & 1446 & 1447 & 1448 & 1449 & 1450 & 1451 & 1452 & 1453 & 1454 \\
\hline 1456 & 1457 & 1458 & 1459 & 1460 & 1461 & 1462 & 1463 & 1464 & 1465 & 1466 & 1467 & 1468 & 1469 \\
\hline 1470 & 1472 & 1473 & 1474 & 1475 & 1476 & 1477 & 1478 & 1479 & 1480 & 1481 & 1482 & 1483 & 1484 \\
\hline 1485 & 1486 & 1488 & 1489 & 1490 & 1491 & 1492 & 1493 & 1494 & 1496 & 1497 & 1498 & 1499 & 1500 \\
\hline 1501 & 1502 & 1504 & 1505 & 1506 & 1507 & 1508 & 1509 & 1510 & 1511 & 1512 & 1513 & 1514 & 1515 \\
\hline 1516 & 1517 & 1518 & 1520 & 1521 & 1522 & 1523 & 1524 & 1525 & 1526 & 1528 & 1529 & 1530 & 1531 \\
\hline 1532 & 1534 & 1632 & 1633 & 1634 & 1635 & 1638 & 1639 & 1640 & 1641 & 1642 & 1643 & 1646 & 1647 \\
\hline 1650 & 1651 & 1652 & 1653 & 1654 & 1656 & 1657 & 1658 & 1659 & 1660 & 1661 & 1662 & 1664 & 1665 \\
\hline 1666 & 1667 & 1668 & 1669 & 1670 & 1671 & 1672 & 1673 & 1674 & 1675 & 1676 & 1677 & 1678 & 1679 \\
\hline 1680 & 1681 & 1682 & 1683 & 1684 & 1685 & 1686 & 1687 & 1688 & 1689 & 1690 & 1691 & 1692 & 1693 \\
\hline 1694 & 1695 & 1696 & 1697 & 1698 & 1699 & 1700 & 1701 & 1702 & 1703 & 1704 & 1705 & 1706 & 1707 \\
\hline 1708 & 1709 & 1710 & 1711 & 1712 & 1713 & 1714 & 1715 & 1716 & 1717 & 1718 & 1719 & 1720 & 1721 \\
\hline 1722 & 1723 & 1724 & 1725 & 1726 & 1728 & 1729 & 1730 & 1731 & 1732 & 1733 & 1734 & 1735 & 1736 \\
\hline 1737 & 1738 & 1739 & 1740 & 1741 & 1742 & 1743 & 1744 & 1745 & 1746 & 1747 & 1748 & 1749 & 1750 \\
\hline 1751 & 1752 & 1753 & 1754 & 1755 & 1756 & 1757 & 1758 & 1760 & 1761 & 1762 & 1763 & 1764 & 1765 \\
\hline 1766 & 1767 & 1768 & 1769 & 1770 & 1771 & 1772 & 1773 & 1774 & 1775 & 1776 & 1777 & 1778 & 1779 \\
\hline 1780 & 1781 & 1782 & 1784 & 1785 & 1786 & 1787 & 1788 & 1789 & 1790 & 1910 & 1912 & 1913 & 1914 \\
\hline 1918 & 1920 & 1921 & 1922 & 1923 & 1924 & 1925 & 1926 & 1928 & 1929 & 1930 & 1931 & 1932 & 1933 \\
\hline 1934 & 1936 & 1937 & 1938 & 1939 & 1940 & 1941 & 1942 & 1944 & 1945 & 1946 & 1947 & 1948 & 1949 \\
\hline 1950 & 1952 & 1953 & 1954 & 1955 & 1956 & 1957 & 1958 & 1960 & 1961 & 1962 & 1963 & 1964 & 1965 \\
\hline
\end{tabular}


Table A.3. Table 1A. (continued).

\begin{tabular}{|c|c|c|c|c|c|c|c|c|c|c|c|c|c|}
\hline 1966 & 1968 & 1969 & 1970 & 1972 & 1973 & 1974 & 1976 & 1977 & 1978 & 1980 & 1982 & 1984 & 1985 \\
\hline 1986 & 1987 & 1988 & 1989 & 1990 & 1992 & 1993 & 1994 & 1995 & 1996 & 1997 & 1998 & 2000 & 2001 \\
\hline 2002 & 2003 & 2004 & 2006 & 2008 & 2009 & 2010 & 2011 & 2012 & 2014 & 2016 & 2017 & 2018 & 2019 \\
\hline 2020 & 2021 & 2022 & 2024 & 2025 & 2026 & 2027 & 2028 & 2029 & 2030 & 2032 & 2033 & 2034 & 2036 \\
\hline 2038 & 2040 & 2041 & 2042 & 2044 & 2046 & 2176 & 2177 & 2178 & 2179 & 2182 & 2183 & 2184 & 2185 \\
\hline 2186 & 2187 & 2190 & 2191 & 2192 & 2193 & 2194 & 2195 & 2196 & 2197 & 2198 & 2199 & 2200 & 2201 \\
\hline 2202 & 2203 & 2204 & 2205 & 2206 & 2208 & 2209 & 2210 & 2211 & 2212 & 2213 & 2214 & 2215 & 2216 \\
\hline 2217 & 2218 & 2219 & 2220 & 2221 & 2222 & 2223 & 2224 & 2225 & 2226 & 2227 & 2228 & 2229 & 2230 \\
\hline 2232 & 2233 & 2234 & 2235 & 2236 & 2237 & 2238 & 2240 & 2241 & 2242 & 2243 & 2244 & 2245 & 2246 \\
\hline 2247 & 2248 & 2249 & 2250 & 2251 & 2252 & 2253 & 2254 & 2255 & 2256 & 2257 & 2258 & 2259 & 2260 \\
\hline 2261 & 2262 & 2263 & 2264 & 2265 & 2266 & 2267 & 2268 & 2269 & 2270 & 2272 & 2273 & 2274 & 2275 \\
\hline 2276 & 2277 & 2278 & 2279 & 2280 & 2281 & 2282 & 2283 & 2284 & 2285 & 2286 & 2287 & 2288 & 2289 \\
\hline 2290 & 2291 & 2292 & 2293 & 2294 & 2296 & 2297 & 2298 & 2299 & 2300 & 2301 & 2302 & 2448 & 2449 \\
\hline 2450 & 2451 & 2454 & 2455 & 2456 & 2457 & 2458 & 2459 & 2462 & 2464 & 2465 & 2466 & 2467 & 2468 \\
\hline 2469 & 2470 & 2471 & 2472 & 2473 & 2474 & 2475 & 2476 & 2477 & 2478 & 2480 & 2481 & 2482 & 2484 \\
\hline 2485 & 2486 & 2488 & 2489 & 2490 & 2492 & 2493 & 2494 & 2496 & 2497 & 2498 & 2499 & 2500 & 2501 \\
\hline 2502 & 2503 & 2504 & 2505 & 2506 & 2507 & 2508 & 2509 & 2510 & 2512 & 2513 & 2514 & 2515 & 2516 \\
\hline 2517 & 2518 & 2519 & 2520 & 2521 & 2522 & 2523 & 2524 & 2525 & 2526 & 2528 & 2529 & 2530 & 2531 \\
\hline 2532 & 2533 & 2534 & 2535 & 2536 & 2537 & 2538 & 2539 & 2540 & 2541 & 2542 & 2544 & 2545 & 2546 \\
\hline 2548 & 2549 & 2550 & 2552 & 2553 & 2554 & 2556 & 2557 & 2558 & 2720 & 2721 & 2722 & 2723 & 2724 \\
\hline 2725 & 2726 & 2727 & 2728 & 2729 & 2730 & 2731 & 2732 & 2733 & 2734 & 2735 & 2736 & 2737 & 2738 \\
\hline 2739 & 2740 & 2741 & 2742 & 2744 & 2745 & 2746 & 2747 & 2748 & 2749 & 2750 & 2752 & 2753 & 2754 \\
\hline 2755 & 2758 & 2759 & 2760 & 2761 & 2762 & 2763 & 2766 & 2767 & 2768 & 2769 & 2770 & 2771 & 2772 \\
\hline 2773 & 2774 & 2775 & 2776 & 2777 & 2778 & 2779 & 2780 & 2781 & 2782 & 2784 & 2785 & 2786 & 2787 \\
\hline 2788 & 2789 & 2790 & 2791 & 2792 & 2793 & 2794 & 2795 & 2796 & 2797 & 2798 & 2800 & 2801 & 2802 \\
\hline 2803 & 2804 & 2805 & 2806 & 2808 & 2809 & 2810 & 2812 & 2813 & 2814 & 2976 & 2977 & 2978 & 2979 \\
\hline 2980 & 2981 & 2982 & 2983 & 2984 & 2985 & 2986 & 2987 & 2988 & 2989 & 2990 & 2992 & 2993 & 2994 \\
\hline 2996 & 2997 & 2998 & 3000 & 3001 & 3002 & 3004 & 3005 & 3006 & 3024 & 3025 & 3026 & 3027 & 3030 \\
\hline 3031 & 3032 & 3033 & 3034 & 3035 & 3038 & 3040 & 3041 & 3042 & 3043 & 3044 & 3045 & 3046 & 3047 \\
\hline 3048 & 3049 & 3050 & 3051 & 3052 & 3053 & 3054 & 3056 & 3057 & 3058 & 3060 & 3061 & 3062 & 3064 \\
\hline 3065 & 3066 & 3068 & 3069 & 3070 & 3232 & 3233 & 3234 & 3235 & 3238 & 3239 & 3240 & 3241 & 3242 \\
\hline 3243 & 3246 & 3248 & 3249 & 3250 & 3251 & 3252 & 3253 & 3254 & 3256 & 3257 & 3258 & 3260 & 3261 \\
\hline 3262 & 3296 & 3297 & 3298 & 3299 & 3300 & 3301 & 3302 & 3303 & 3304 & 3305 & 3306 & 3307 & 3308 \\
\hline 3309 & 3310 & 3312 & 3313 & 3314 & 3315 & 3316 & 3317 & 3318 & 3320 & 3321 & 3322 & 3324 & 3325 \\
\hline 3326 & 3504 & 3505 & 3506 & 3510 & 3512 & 3513 & 3514 & 3518 & 3552 & 3553 & 3554 & 3555 & 3556 \\
\hline 3557 & 3558 & 3560 & 3561 & 3562 & 3563 & 3564 & 3566 & 3568 & 3569 & 3570 & 3572 & 3574 & 3576 \\
\hline
\end{tabular}


Table A.4. Table 1A. (continued).

\begin{tabular}{|c|c|c|c|c|c|c|c|c|c|c|c|c|c|}
\hline 3577 & 3578 & 3580 & 3582 & 3808 & 3809 & 3810 & 3811 & 3814 & 3815 & 3816 & 3817 & 3818 & 3819 \\
\hline 3822 & 3824 & 3825 & 3826 & 3828 & 3829 & 3830 & 3832 & 3833 & 3834 & 3836 & 3837 & 3838 & 4080 \\
\hline 4081 & 4082 & 4086 & 4088 & 4089 & 4090 & 4094 & 5160 & 5161 & 5162 & 5163 & 5166 & 180 & 5181 \\
\hline 5182 & 5224 & 5225 & 5226 & 5227 & 5228 & 5229 & 5230 & 5244 & 5245 & 5246 & 5248 & 5249 & 5250 \\
\hline 5251 & 5252 & 5253 & 5254 & 5255 & 5256 & 5257 & 5258 & 5259 & 5260 & 5261 & 5262 & 5268 & 5269 \\
\hline 5270 & 5271 & 5272 & 5273 & 5274 & 5275 & 5276 & 5277 & 5278 & 5288 & 5289 & 5290 & 5291 & 5292 \\
\hline 5293 & 5294 & 5308 & 5309 & 5310 & 5312 & 5313 & 5314 & 5315 & 5316 & 5317 & 5318 & 5319 & 5320 \\
\hline 5321 & 5322 & 5323 & 5324 & 5325 & 5326 & 5332 & 5333 & 5334 & 5335 & 5336 & 5337 & 5338 & 5339 \\
\hline 5340 & 5341 & 5342 & 5352 & 5353 & 5354 & 5355 & 5356 & 5357 & 5358 & 5372 & 5373 & 5374 & 5438 \\
\hline 5482 & 5483 & 5486 & 5502 & 5504 & 5505 & 5506 & 5507 & 5508 & 5510 & 5512 & 5513 & 5514 & 5515 \\
\hline 5516 & 5518 & 5524 & 5526 & 5528 & 5530 & 5532 & 5534 & 5544 & 5545 & 5546 & 5547 & 5548 & 5550 \\
\hline 5564 & 5566 & 5568 & 5569 & 5570 & 5571 & 5572 & 5574 & 5576 & 5577 & 5578 & 5579 & 5580 & 5582 \\
\hline 5588 & 5590 & 5592 & 5594 & 5596 & 5598 & 5608 & 5609 & 5610 & 5611 & 5612 & 5614 & 5628 & 5630 \\
\hline 5736 & 5737 & 5738 & 5739 & 5742 & 5756 & 5757 & 5758 & 5760 & 5761 & 5762 & 5763 & 5764 & 5765 \\
\hline 5766 & 5767 & 5768 & 5769 & 5770 & 5771 & 5772 & 5773 & 5774 & 5780 & 5781 & 5782 & 5783 & 5784 \\
\hline 5785 & 5786 & 5787 & 5788 & 5789 & 5790 & 5800 & 5801 & 5802 & 5803 & 5804 & 5805 & 5806 & 5820 \\
\hline 5821 & 5822 & 5824 & 5825 & 5826 & 5827 & 5828 & 5829 & 5830 & 5831 & 5832 & 5833 & 5834 & 5835 \\
\hline 5836 & 5837 & 5838 & 5844 & 5845 & 5846 & 5848 & 5849 & 5850 & 5851 & 5852 & 5853 & 5854 & 5864 \\
\hline 5865 & 5866 & 5867 & 5868 & 5869 & 5870 & 5884 & 5885 & 5886 & 6014 & 6016 & 6017 & 6018 & 6019 \\
\hline 6020 & 6022 & 6024 & 6025 & 6026 & 6027 & 6028 & 6030 & 6036 & 6038 & 6040 & 6042 & 6044 & 6046 \\
\hline 6056 & 6057 & 6058 & 6059 & 6060 & 6062 & 6076 & 6078 & 6080 & 6081 & 6082 & 6083 & 6084 & 6086 \\
\hline 6088 & 6089 & 6090 & 6091 & 6092 & 6094 & 6100 & 6102 & 6104 & 6106 & 6108 & 6110 & 6120 & 6121 \\
\hline 6122 & 6123 & 6124 & 6126 & 6140 & 6142 & 6272 & 6273 & 6274 & 6275 & 6278 & 6279 & 6280 & 6281 \\
\hline 6282 & 6283 & 6286 & 6288 & 6289 & 6290 & 6291 & 6292 & 6293 & 6294 & 6296 & 6297 & 6298 & 6299 \\
\hline 6300 & 6301 & 6302 & 6304 & 6305 & 6306 & 6307 & 6308 & 6309 & 6310 & 6311 & 6312 & 6313 & 6314 \\
\hline 6315 & 6316 & 6317 & 6318 & 6320 & 6321 & 6322 & 6324 & 6325 & 6326 & 6328 & 6329 & 6330 & 6332 \\
\hline 6333 & 6334 & 6336 & 6337 & 6338 & 6339 & 6340 & 6341 & 6342 & 6343 & 6344 & 6345 & 6346 & 6347 \\
\hline 6348 & 6349 & 6350 & 6352 & 6353 & 6354 & 6355 & 6356 & 6357 & 6358 & 6360 & 6361 & 6362 & 6363 \\
\hline 6364 & 6365 & 6366 & 6368 & 6369 & 6370 & 6371 & 6372 & 6373 & 6374 & 6375 & 6376 & 6377 & 6378 \\
\hline 6379 & 6380 & 6381 & 6382 & 6384 & 6385 & 6386 & 6388 & 6389 & 6390 & 6392 & 6393 & 6394 & 6396 \\
\hline 6397 & 6398 & 6544 & 6546 & 6550 & 6552 & 6554 & 6558 & 6560 & 6561 & 6562 & 6563 & 6564 & 6565 \\
\hline 6566 & 6568 & 6569 & 6570 & 6571 & 6572 & 6573 & 6574 & 6576 & 6578 & & 6582 & 6584 & 6586 \\
\hline 6588 & 6590 & 6592 & 6593 & 6594 & 6595 & 6596 & 6597 & & 6600 & & & 6603 & 6604 \\
\hline 6605 & 6606 & 6608 & 6610 & 6612 & 6614 & 6616 & 6618 & 6620 & & 6624 & & 6626 & 6627 \\
\hline 6628 & 6629 & 6630 & 6632 & 6633 & 6634 & 6635 & 6636 & 6638 & 6640 & 6642 & 6644 & 6646 & 6648 \\
\hline 6650 & 6652 & 6654 & 6816 & 6817 & 6818 & 6819 & 6820 & 6821 & 6822 & 6823 & 6824 & 6825 & 6826 \\
\hline
\end{tabular}


Table A.5. Table 1A. (continued).

\begin{tabular}{|c|c|c|c|c|c|c|c|c|c|c|c|c|c|}
\hline 6827 & 6828 & 6829 & 6830 & 6832 & 6833 & 6834 & 6836 & 6837 & 6838 & 6840 & 6841 & 6842 & 6844 \\
\hline 6845 & 6846 & 6848 & 6849 & 6850 & 6851 & 6854 & 6855 & 6856 & 6857 & 6858 & 6859 & 6862 & 6864 \\
\hline 6865 & 6866 & 6868 & 6869 & 6870 & 6872 & 6873 & 6874 & 6876 & 6877 & 6878 & 6880 & 6881 & 6882 \\
\hline 6883 & 6884 & 6885 & 6886 & 6888 & 6889 & 6890 & 6891 & 6892 & 6893 & 6894 & 6896 & 6897 & 6898 \\
\hline 6900 & 6901 & 6902 & 6904 & 6905 & 6906 & 6908 & 6909 & 6910 & 7072 & 7073 & 7074 & 7075 & 7076 \\
\hline 7077 & 7078 & 7080 & 7081 & 7082 & 7083 & 7084 & 7086 & 7088 & 7090 & 7092 & 7094 & 7096 & 7098 \\
\hline 7100 & 7102 & 7120 & 7122 & 7126 & 7128 & 7130 & 7134 & 7136 & 7137 & 7138 & 7139 & 7140 & 7142 \\
\hline 7144 & 7145 & 7146 & 7147 & 7148 & 7150 & 7152 & 7154 & 7156 & 7158 & 7160 & 7162 & 7164 & 7166 \\
\hline 7328 & 7329 & 7330 & 7331 & 7334 & 7336 & 7337 & 7338 & 7339 & 7342 & 7344 & 7345 & 7346 & 7348 \\
\hline 7349 & 7350 & 7352 & 7353 & 7354 & 7356 & 7357 & 7358 & 7392 & 7393 & 7394 & 7395 & 7396 & 7397 \\
\hline 7398 & 7400 & 7401 & 7402 & 7403 & 7404 & 7405 & 7406 & 7408 & 7409 & 7410 & 7412 & 7413 & 7414 \\
\hline 7416 & 7417 & 7418 & 7420 & 7421 & 7422 & 7600 & 7602 & 7606 & 7608 & 7610 & 7614 & 7648 & 7649 \\
\hline 7650 & 7651 & 7652 & 7654 & 7656 & 7657 & 7658 & 7659 & 7660 & 7662 & 7664 & 7666 & 7668 & 7670 \\
\hline 7672 & 7674 & 7676 & 7678 & 7904 & 7905 & 7906 & 7907 & 7910 & 7912 & 7913 & 7914 & 7915 & 7918 \\
\hline 7920 & 7921 & 7922 & 7924 & 7925 & 7926 & 7928 & 7929 & 7930 & 7932 & 7933 & 7934 & 8176 & 8178 \\
\hline 8182 & 8184 & 8186 & 8190 & 10240 & 10241 & 10242 & 10243 & 10244 & 10245 & 10246 & 10248 & 10249 & 10250 \\
\hline 10251 & 10252 & 10253 & 10254 & 10260 & 10261 & 10262 & 10264 & 10265 & 10266 & 10268 & 10269 & 10270 & 10280 \\
\hline 10281 & 10282 & 10283 & 10284 & 10285 & 10286 & 10300 & 10301 & 10302 & 10304 & 10305 & 10306 & 10307 & 10308 \\
\hline 10309 & 10310 & 10312 & 10313 & 10314 & 10315 & 10316 & 10317 & 10318 & 10324 & 10325 & 10326 & 10328 & 10329 \\
\hline 10330 & 10332 & 10333 & 10334 & 10344 & 10345 & 10346 & 10347 & 10348 & 10349 & 10350 & 10364 & 10365 & 10366 \\
\hline 10368 & 10369 & 10370 & 10371 & 10372 & 10373 & 10374 & 10376 & 10377 & 10378 & 10379 & 10380 & 10381 & 10382 \\
\hline 10388 & 10389 & 10390 & 10392 & 10393 & 10394 & 10396 & 10397 & 10398 & 10408 & 10409 & 10410 & 10411 & 10412 \\
\hline 10413 & 10414 & 10428 & 10429 & 10430 & 10432 & 10433 & 10434 & 10435 & 10436 & 10437 & 10438 & 10440 & 10441 \\
\hline 10442 & 10443 & 10444 & 10445 & 10446 & 10452 & 10453 & 10454 & 10456 & 10457 & 10458 & 10460 & 10461 & 10462 \\
\hline 10472 & 10473 & 10474 & 10475 & 10476 & 10477 & 10478 & 10492 & 10493 & 10494 & 10498 & 10499 & 10502 & 10504 \\
\hline 10505 & 10506 & 10507 & 10508 & 10510 & 10518 & 10520 & 10522 & 10524 & 10526 & 10536 & 10537 & 10538 & 10539 \\
\hline 10540 & 10542 & 10556 & 10558 & 10562 & 10563 & 10566 & 10568 & 10569 & 10570 & 10571 & 10572 & 10574 & 10582 \\
\hline 10584 & 10586 & 10588 & 10590 & 10600 & 10601 & 10602 & 10603 & 10604 & 10606 & 10620 & 10622 & 10624 & 10625 \\
\hline 10626 & 10627 & 10628 & 10630 & 10632 & 10633 & 10634 & 10635 & 10636 & 10638 & 10644 & 10646 & 10648 & 10650 \\
\hline 10652 & 10654 & 10664 & 10665 & 10666 & 10667 & 10668 & 10670 & 10684 & 10686 & 10688 & 10689 & 10690 & 10691 \\
\hline 10692 & 10694 & 10696 & 10697 & 10698 & 10699 & 10700 & 10702 & 10708 & 10710 & 10712 & 10714 & 10716 & 10718 \\
\hline 10728 & 10729 & 10730 & 10732 & 10734 & 10748 & 10750 & 10752 & 10753 & 10754 & 10755 & 10756 & 10757 & 10758 \\
\hline 10760 & 10761 & 10762 & 10763 & 10764 & 10765 & 10766 & 10772 & 10773 & 10774 & 10776 & 10777 & 10778 & 10780 \\
\hline 10781 & 10782 & 10792 & 10793 & 10794 & 10795 & 10796 & 10797 & 10798 & 10812 & 10813 & 10814 & 10816 & 10817 \\
\hline 10818 & 10819 & 10820 & 10821 & 10822 & 10824 & 10825 & 10826 & 10827 & 10828 & 10829 & 10830 & 10836 & 10837 \\
\hline 10838 & 10840 & 10841 & 10842 & 10844 & 10845 & 10846 & 10856 & 10857 & 10858 & 10860 & 10861 & 10862 & 10876 \\
\hline
\end{tabular}


Table A.6. Table 1A. (continued).

\begin{tabular}{|c|c|c|c|c|c|c|c|c|c|c|c|c|}
\hline 10877 & 10878 & 10880 & 10881 & 10882 & 10883 & 10884 & 10885 & 10886 & 10888 & 10889 & 10890 & 10891 \\
\hline 10892 & 10893 & 10894 & 10900 & 10901 & 10902 & 10904 & 10905 & 10906 & 10908 & 10909 & 10910 & 10920 \\
\hline 10921 & 10922 & 10923 & 10924 & 10925 & 10926 & 10940 & 10941 & 10942 & 10944 & 10945 & 10946 & 10947 \\
\hline 10948 & 10949 & 10950 & 10952 & 10953 & 10954 & 10955 & 10956 & 10957 & 10958 & 10964 & 10965 & 10966 \\
\hline 10968 & 10969 & 10970 & 10972 & 10973 & 10974 & 10984 & 10985 & 10986 & 10988 & 10989 & 10990 & 11004 \\
\hline 11005 & 11006 & 11010 & 11011 & 11014 & 11016 & 11017 & 11018 & 11019 & 11020 & 11022 & 11030 & 11032 \\
\hline 11034 & 11036 & 11038 & 11048 & 11049 & 11050 & 11051 & 11052 & 11054 & 11068 & 11070 & 11074 & 11075 \\
\hline 11078 & 11080 & 11081 & 11082 & 11083 & 11084 & 11086 & 11094 & 11096 & 11098 & 11100 & 11102 & 11112 \\
\hline 11113 & 11114 & 11116 & 11118 & 11132 & 11134 & 11136 & 11137 & 11138 & 11139 & 11140 & 11142 & 11144 \\
\hline 11145 & 11146 & 11147 & 11148 & 11150 & 11156 & 11158 & 11160 & 11162 & 11164 & 11166 & 11176 & 11177 \\
\hline 11178 & 11180 & 11182 & 11196 & 11198 & 11200 & 11201 & 11202 & 11203 & 11204 & 11206 & 11208 & 11209 \\
\hline 11210 & 11211 & 11212 & 11214 & 11220 & 11222 & 11224 & 11226 & 11228 & 11230 & 11240 & 11241 & 11242 \\
\hline 11244 & 11246 & 11260 & 11262 & 11272 & 11273 & 11274 & 11275 & 11276 & 11278 & 11288 & 11289 & 11290 \\
\hline 11292 & 11294 & 11296 & 11297 & 11298 & 11299 & 11300 & 11301 & 11302 & 11304 & 11305 & 11306 & 11308 \\
\hline 11310 & 11314 & 11316 & 11317 & 11318 & 11320 & 11321 & 11322 & 11324 & 11326 & 11336 & 11337 & 11338 \\
\hline 11339 & 11340 & 11342 & 11352 & & 11354 & 11356 & 11358 & 11360 & 11361 & 11362 & 11363 & 11364 \\
\hline 11365 & 11366 & & & & & & & 11380 & 11381 & & & 11385 \\
\hline 11386 & 11388 & 11390 & & & & & & 11397 & 11398 & & & 11402 \\
\hline 11403 & 11404 & 11406 & & 11409 & 11410 & & 11413 & 11414 & 11416 & 11417 & 11418 & 11420 \\
\hline 11422 & 11424 & 11425 & 11426 & 11427 & 11428 & 11429 & 11430 & 11432 & 11433 & 11434 & 11436 & 11438 \\
\hline 11440 & 11441 & 11442 & 11444 & 11445 & 11446 & 11448 & 11449 & 11450 & 11452 & 11454 & 11456 & 11457 \\
\hline 11458 & 11459 & 11460 & 11461 & 11462 & 11464 & 11465 & 11466 & 11467 & 11468 & 11470 & 11472 & 11473 \\
\hline 11474 & 11476 & 11477 & 11478 & 11480 & 11481 & 11482 & 11484 & 11486 & 11488 & 11489 & 11490 & 11491 \\
\hline 11492 & 11493 & 11494 & 11496 & 11497 & 11498 & 11500 & 11502 & 11504 & 11505 & 11506 & 11508 & 11509 \\
\hline 11510 & 11512 & 11513 & 11514 & 11516 & 11518 & 11530 & 11531 & 11534 & 11546 & 11550 & 11554 & 11555 \\
\hline 11558 & 11560 & 11561 & 11562 & 11564 & 11566 & 11574 & 11576 & 11578 & 11580 & 11582 & 11594 & 11595 \\
\hline 11598 & 11610 & 11614 & 11618 & 11619 & 11622 & 11624 & 11625 & 11626 & 11628 & 11630 & 11638 & 11640 \\
\hline 11642 & 11644 & 11646 & 11648 & 11649 & 11650 & 11651 & 11652 & 11654 & 11656 & 11657 & 11658 & 11659 \\
\hline 11660 & 11662 & 11664 & 11666 & 11668 & 11670 & 11672 & 11674 & 11676 & 11678 & 11680 & 11681 & 11682 \\
\hline 11683 & 11684 & 11686 & 11688 & 11689 & 11690 & 11692 & 11694 & 11696 & 11698 & 11700 & 11702 & 11704 \\
\hline 11706 & 11708 & 11710 & 11712 & 11713 & 11714 & 11716 & 11718 & 11720 & 11721 & 11722 & 11724 & 11726 \\
\hline 11728 & 11730 & 11732 & 11734 & & 11738 & & 11742 & 11744 & 11745 & 11746 & 11748 & 11750 \\
\hline 11752 & & 11756 & 11758 & 11760 & 11762 & 11764 & 11766 & 11768 & 11770 & 11772 & 11774 & 11784 \\
\hline 11785 & 11786 & 11787 & 11788 & 11750 & 11800 & 11001 & 11802 & 11804 & 11806 & 11808 & 11809 & 11810 \\
\hline 11811 & 11812 & 11814 & 11816 & 11817 & 11818 & 11820 & 11822 & 11826 & 11828 & 11830 & 11832 & 11834 \\
\hline 11836 & 11838 & 11848 & 11849 & 11850 & 11852 & 11854 & 11864 & 11865 & 11866 & 11868 & 11870 & 11872 \\
\hline
\end{tabular}


Table A.7. Table 1A. (continued).

\begin{tabular}{|c|c|c|c|c|c|c|c|c|c|c|c|c|}
\hline 1873 & 1874 & 11876 & 11878 & 1880 & 11881 & 11882 & 11884 & 11886 & 11890 & 11892 & 11894 & 1896 \\
\hline 11898 & 11900 & 11902 & 11904 & 11905 & 11906 & 11907 & 11908 & 11910 & 11912 & 1913 & 11914 & 1915 \\
\hline 916 & 1918 & 11920 & 1921 & 1922 & 11924 & 11926 & 11928 & & 11930 & 1932 & 1934 & 1936 \\
\hline 1937 & 1938 & 11939 & 11940 & 1942 & 11944 & 11945 & 11946 & 11948 & 11950 & 1952 & 11954 & 11956 \\
\hline 11958 & 1960 & 11962 & 11964 & 1966 & 11968 & 11969 & 11970 & 11971 & 11972 & 11974 & & 11977 \\
\hline 11978 & 11980 & 11982 & 11984 & 11985 & 11986 & 11988 & 11990 & 11992 & 11993 & 11994 & & 11998 \\
\hline 12000 & 12001 & 12002 & 12004 & 12006 & 12008 & 12009 & 12010 & 12012 & 12014 & 12016 & 12018 & 12020 \\
\hline 12022 & 12024 & 12026 & 12028 & 12030 & 12042 & 12043 & 12046 & 12058 & 12062 & 12066 & 12067 & 12070 \\
\hline 12072 & 12073 & 12074 & 12076 & 12078 & 12086 & 12088 & 12090 & 12092 & 12094 & 12106 & & 12122 \\
\hline 12126 & 12130 & 12134 & 12136 & 12138 & 12140 & 12142 & 12150 & 12152 & 12154 & 12156 & 12158 & 12160 \\
\hline 12161 & 12162 & 12164 & 12166 & 12168 & 12169 & 12170 & 12172 & 12174 & 12176 & 12178 & 12180 & 12182 \\
\hline 12184 & 12186 & 12188 & 12190 & 12192 & 12193 & 12194 & 12196 & 12198 & 12200 & 12202 & 12204 & 12206 \\
\hline 12208 & 12210 & 12212 & 12214 & 12216 & 12218 & 12220 & 12222 & 12224 & 12226 & 12228 & 12230 & 12232 \\
\hline 12234 & 12236 & 12238 & 12240 & 12242 & 12244 & 12246 & 12248 & 12250 & 12252 & 12254 & 12256 & 12258 \\
\hline 12260 & 12262 & 12264 & 12266 & 12268 & 12270 & 12272 & 12274 & 12276 & 12278 & 12280 & 12282 & 12284 \\
\hline 12286 & 15400 & 15401 & 15402 & 15404 & 15406 & 15420 & 15422 & 15464 & 15465 & 15466 & 15468 & 15470 \\
\hline 15484 & 15486 & 15488 & 15489 & 15490 & 15491 & 15492 & 15494 & 15496 & 15497 & 15498 & 15500 & 15502 \\
\hline 15508 & 15510 & 15512 & 15514 & 15516 & 15518 & 15528 & 15529 & 15530 & 15532 & 15534 & 15548 & 15550 \\
\hline 15552 & 15553 & 15554 & 15555 & 15556 & 15558 & 15560 & 15561 & 15562 & 15564 & & & 15574 \\
\hline 15576 & 15578 & 15580 & 15582 & 15592 & 15593 & 15594 & 15596 & 15598 & 15612 & 15614 & 15658 & 15662 \\
\hline 15678 & 15722 & 15726 & 15742 & 15744 & 15746 & 15748 & 15750 & 15752 & 15754 & 15756 & & 15764 \\
\hline 15766 & 15768 & 15770 & 15772 & 15774 & 15784 & 15786 & 15788 & 15790 & 15804 & & & 15810 \\
\hline 15812 & 15814 & 15816 & 15818 & 15820 & 15822 & 15828 & 15830 & 15832 & & & & 15848 \\
\hline 15850 & 15852 & 15854 & 15868 & 15870 & 15912 & 15913 & 15914 & 15916 & & & & 15976 \\
\hline 15977 & 15978 & 15980 & 15982 & 15996 & 15998 & 16000 & 16001 & 16002 & & 16004 & & 16008 \\
\hline 16009 & 16010 & 16012 & 16014 & 16020 & 16022 & 16024 & 16026 & 16028 & & 16040 & & 16042 \\
\hline 16044 & 16046 & 16060 & 16062 & 16064 & 16065 & 16066 & 16068 & 16070 & & 16073 & & 16076 \\
\hline 16078 & 16084 & 16086 & 16088 & 16090 & 16092 & 16094 & 16104 & 16105 & & 16108 & & 16124 \\
\hline 16126 & 16170 & 16174 & 16190 & 16234 & 16238 & 16254 & 16256 & 16258 & & & & 16266 \\
\hline 16268 & 16270 & 16276 & 16278 & 16280 & 16282 & 16284 & & & & & & 16316 \\
\hline 16318 & 16320 & 16322 & 16324 & 16326 & 16328 & & & & & & & 16346 \\
\hline 16348 & 16350 & & & & & & & & & & & 26760 \\
\hline 26761 & 26762 & 26766 & 26772 & 26774 & & 26778 & & & & & & 26796 \\
\hline 26798 & 26812 & 26814 & & 26817 & & & & & & & & 26830 \\
\hline 26836 & 26838 & 26840 & 26842 & 26844 & 26846 & 26856 & 26857 & & 26860 & & 26876 & 26878 \\
\hline 27030 & 27032 & 27034 & 27038 & 27048 & 27050 & 27052 & 27054 & 27068 & 27070 & 27074 & 27078 & 27080 \\
\hline
\end{tabular}


Table A.8. Table 1A. (continued).

\begin{tabular}{|c|c|c|c|c|c|c|c|c|c|c|c|c|}
\hline 27082 & 27084 & 27086 & 27094 & 27096 & 27098 & 27100 & 27102 & 27112 & 27114 & 27116 & 27118 & 27132 \\
\hline 27134 & 27304 & 27305 & 27306 & 27308 & 27310 & 27324 & 27326 & 27328 & 27329 & 27330 & 27334 & 27336 \\
\hline 27337 & 27338 & 27342 & 27348 & 27350 & 27352 & 27354 & 27356 & 27358 & 27368 & 27370 & 27372 & 27374 \\
\hline 27388 & 27390 & 27560 & 27562 & 27564 & 27566 & 27580 & 27582 & 27606 & 27608 & 27610 & 27614 & 27624 \\
\hline 27626 & 27628 & 27630 & 27644 & 27646 & 27808 & 27809 & 27810 & 27814 & 27816 & 27818 & 27822 & 27826 \\
\hline 27828 & 27830 & 27832 & 27834 & 27836 & 27838 & 27872 & 27873 & 27874 & 27876 & 27878 & 27880 & 27882 \\
\hline 27884 & 27886 & 27890 & 27892 & 27894 & 27896 & 27898 & 27900 & 27902 & 28086 & 28088 & 28090 & 28094 \\
\hline 28130 & 28134 & 28136 & 28138 & 28140 & 28142 & 28150 & 28152 & 28154 & 28156 & 28158 & 28384 & 28386 \\
\hline 28390 & 28392 & 28394 & 28398 & 28402 & 28404 & 28406 & 28408 & 28410 & 28412 & 28414 & 28662 & 28664 \\
\hline 28666 & 28670 & 31912 & 31914 & 31918 & 31932 & 31934 & 31976 & 31978 & 31980 & 31982 & 31996 & 31998 \\
\hline 32190 & 32234 & 32238 & 32254 & 32488 & 32490 & 32494 & 32508 & 32510 & 32766 & 32768 & 32770 & 32774 \\
\hline 32776 & 32778 & 32782 & 32788 & 32790 & 32792 & 32794 & 32796 & 32798 & 32808 & 32810 & 32812 & 32814 \\
\hline 32828 & 32830 & 32832 & 32834 & 32836 & 32838 & 32840 & 32842 & 32844 & 32846 & 32852 & 32854 & 32856 \\
\hline 32858 & 32860 & 32862 & 32872 & 32874 & 32876 & 32878 & 32892 & 32894 & 32896 & 32898 & 32900 & 32902 \\
\hline 32904 & 32906 & 32908 & 32910 & 32916 & 32918 & 32920 & 32922 & 32924 & 32926 & 32936 & 32938 & 32940 \\
\hline 32942 & 32956 & 32958 & 32960 & 32962 & 32964 & 32966 & 32968 & 32970 & 32972 & 32974 & 32980 & 32982 \\
\hline 32984 & 32986 & 32988 & 32990 & 33000 & 33002 & 33004 & 33006 & 33020 & 33022 & 33046 & 33048 & 33050 \\
\hline 33054 & 33064 & 33066 & 33068 & 33070 & 33084 & 33086 & 33090 & 33094 & 33096 & 33098 & 33100 & 33102 \\
\hline 33110 & 33112 & 33114 & 33116 & 33118 & 33128 & 33130 & 33132 & 33134 & 33148 & 33150 & 33152 & 33154 \\
\hline 33156 & 33158 & 33160 & 33162 & 33164 & 33166 & 33172 & 33174 & 33176 & 33178 & 33180 & 33182 & 33192 \\
\hline 33194 & 33196 & 33198 & 33212 & 33214 & 33216 & 33218 & 33220 & 33222 & 33224 & 33226 & 33228 & 33230 \\
\hline 33236 & 33238 & 33240 & 33242 & 33244 & 33246 & 33256 & 33258 & 33260 & 33262 & 33276 & 33320 & 33322 \\
\hline 33324 & 33326 & 33340 & 33342 & 33344 & 33346 & 33350 & 33352 & 33354 & 33358 & 33364 & 33366 & 33368 \\
\hline 33370 & 33372 & 33374 & 33384 & 33386 & 33388 & 33390 & 33404 & 33408 & 33410 & 33412 & 33414 & 33416 \\
\hline 33418 & 33420 & 33422 & 33428 & 33430 & 33432 & 33434 & 33436 & 33438 & 33448 & 33450 & 33452 & 33454 \\
\hline 33468 & 33470 & 33472 & 33474 & 33476 & 33478 & 33480 & 33482 & 33484 & 33486 & 33492 & 33494 & 33496 \\
\hline 33498 & 33500 & 33502 & 33512 & 33514 & 33516 & 33518 & 33532 & 33576 & 33578 & 33580 & 33582 & 33596 \\
\hline 33598 & 33622 & 33624 & 33626 & 33630 & 33640 & 33642 & 33644 & 33646 & 33660 & 33664 & 33666 & 33668 \\
\hline 33670 & 33672 & 33674 & 33676 & 33678 & 33684 & 33686 & 33688 & 33690 & 33692 & 33694 & 33704 & 33706 \\
\hline 33708 & 33710 & 33724 & 33728 & 33730 & 33732 & 33734 & 33736 & 33738 & 33740 & 33742 & 33748 & 33750 \\
\hline 33752 & 33754 & 33756 & 33758 & 33768 & 33770 & 33772 & 33774 & 33788 & 33824 & 33826 & 33830 & 33832 \\
\hline 33834 & 33838 & 33842 & 33844 & 33846 & 33848 & 33850 & 33852 & 33888 & 33890 & 33892 & 33894 & 33896 \\
\hline 33898 & 33900 & 33902 & 33906 & 33908 & 33910 & 33912 & 33914 & 33916 & 33920 & 33922 & 33924 & 33926 \\
\hline 33928 & 33930 & 33932 & 33934 & 33936 & 33938 & 33940 & 33942 & 33944 & 33946 & 33948 & 33950 & 33952 \\
\hline 33954 & 33956 & 33958 & 33960 & 33962 & 33964 & 33966 & 33968 & 33970 & 33972 & 33974 & 33976 & 33978 \\
\hline 33980 & 33984 & 33986 & 33988 & 33990 & 33992 & 33994 & 33996 & 33998 & 34000 & 34002 & 34004 & 34006 \\
\hline
\end{tabular}


Table A.9. Table 1A. (continued).

\begin{tabular}{|c|c|c|c|c|c|c|c|c|c|c|c|c|}
\hline 34008 & 4010 & 4012 & 4014 & 34016 & 34018 & 34020 & 34022 & 34024 & 34026 & 34028 & 34030 & 34032 \\
\hline 34034 & 34036 & 34038 & 34040 & 34042 & 34044 & 34102 & 34104 & 34106 & 34146 & 34150 & 34152 & 34154 \\
\hline 34156 & 34166 & 34168 & 34170 & 34172 & 34176 & 34178 & 34180 & 34182 & 34184 & 34186 & 34188 & 34192 \\
\hline 34194 & 34196 & 34198 & 34200 & 34202 & 34204 & 34208 & 34210 & 34212 & 34214 & 34216 & 34218 & 34220 \\
\hline 34224 & 34226 & 34228 & 34230 & 34232 & 34234 & 34236 & 34240 & 34242 & 34244 & 34246 & 34248 & 34250 \\
\hline 34252 & 34256 & 34258 & 34260 & 34264 & 34266 & 34268 & 34272 & 34274 & 34276 & 34278 & 34280 & 34282 \\
\hline 34284 & 34288 & 34290 & 34292 & 34296 & 34298 & 34400 & 34402 & 34406 & 34408 & 34410 & 34414 & 34418 \\
\hline 34420 & 34424 & 34426 & 34428 & 34432 & 34434 & 34436 & 34438 & 34440 & 34442 & 34444 & 34446 & 34448 \\
\hline 34450 & 34452 & 34454 & 34456 & 34458 & 34460 & 34462 & 34464 & 34466 & 34468 & 34470 & 34472 & 34474 \\
\hline 34476 & 34478 & 34480 & 34482 & 34484 & 34486 & 34488 & 34490 & 34492 & 34496 & 34498 & 34500 & 34502 \\
\hline 34504 & 34506 & 34508 & 34510 & 34512 & 34514 & 34516 & 34518 & 34520 & 34522 & 34524 & 34528 & 34530 \\
\hline 34532 & 34534 & 34536 & 34538 & 34540 & 34542 & 34544 & 34546 & 34548 & 34552 & 34554 & 34556 & 34680 \\
\hline 34688 & 34690 & 34692 & 34696 & 34698 & 34700 & 34704 & 34706 & 34708 & 34712 & 34714 & 34716 & 34720 \\
\hline 34722 & 34724 & 34728 & 34730 & 34732 & 34736 & 34740 & 34744 & 34752 & 34754 & 34756 & 34760 & 34762 \\
\hline 34764 & 34768 & 34770 & 34776 & 34778 & 34784 & 34786 & 34788 & 34792 & 34794 & 34796 & 34800 & 34808 \\
\hline 34944 & 34946 & 34950 & 34952 & 34954 & 34958 & 34960 & 34962 & 34964 & 34966 & 34968 & 34970 & 34972 \\
\hline 34976 & 34978 & 34980 & 34982 & 34984 & 34986 & 34988 & 34990 & 34992 & 34994 & 34996 & 35000 & 35002 \\
\hline 35004 & 35008 & 35010 & 35012 & 35014 & 35016 & 35018 & 35020 & 35022 & 35024 & 35026 & 35028 & 35030 \\
\hline 35032 & 35034 & 35036 & 35040 & 35042 & 35044 & 35046 & 35048 & 35050 & 35052 & 35054 & 35056 & 35058 \\
\hline 35060 & 35064 & 35066 & 35068 & 35216 & 35218 & 35222 & 35224 & 35226 & 35232 & 35234 & 35236 & 35238 \\
\hline 35240 & 35242 & 35244 & 35248 & 35252 & 35256 & 35260 & 35264 & 35266 & 35268 & 35270 & 35272 & 35274 \\
\hline 35276 & 35280 & 35282 & 35284 & 35286 & 35288 & 35290 & 35292 & 35296 & 35298 & 35300 & 35302 & 35304 \\
\hline 35306 & 35308 & 35312 & 35316 & 35320 & 35324 & 35488 & 35490 & 35492 & 35494 & 35496 & 35498 & 35500 \\
\hline 35502 & 35504 & 35506 & 35508 & 35512 & 35514 & 35516 & 35520 & 35522 & 35526 & 35528 & 35530 & 35534 \\
\hline 35536 & 35538 & 35540 & 35542 & 35544 & 35546 & 35548 & 35552 & 35554 & 35556 & 35558 & 35560 & 35562 \\
\hline 35564 & 35568 & 35570 & 35572 & 35576 & & 35744 & 35746 & 35748 & 35750 & & 35754 & 35756 \\
\hline 35760 & 35764 & 35768 & 35772 & 35792 & 35794 & 35798 & & & & & 35812 & 35814 \\
\hline 35816 & 35818 & 35820 & 35824 & 35828 & 35832 & 35836 & 36000 & & & & & 36016 \\
\hline 36018 & 36020 & 36024 & 36028 & 36064 & 36066 & 36068 & 36070 & 36072 & & & 36080 & 36082 \\
\hline 36084 & 36088 & 36092 & 36272 & 36280 & 36320 & 36322 & 36324 & 36328 & 36330 & 36336 & 36344 & 36576 \\
\hline 36578 & 36582 & & 36586 & & 36596 & & 36604 & 36848 & 36856 & 37928 & 37930 & 37948 \\
\hline 37992 & 37994 & 37996 & 38012 & 38016 & 38018 & 38020 & 38022 & 38024 & & 38028 & 38036 & 38038 \\
\hline 38040 & 38042 & 38044 & 38056 & 38058 & 38060 & 38076 & 38080 & 38082 & 38084 & 38086 & 38088 & 38090 \\
\hline 38092 & 38100 & 38102 & 38104 & 38106 & 38108 & 38120 & 38122 & 38124 & 38140 & 38250 & 38272 & 38274 \\
\hline 38280 & 38282 & 38312 & 38314 & 38336 & 38338 & 38344 & 38346 & 38376 & 38378 & 38504 & 38506 & 38524 \\
\hline 38528 & 38530 & 38532 & 38534 & 38536 & 38538 & 38540 & 38548 & 38550 & 38552 & 38554 & 38556 & 38568 \\
\hline
\end{tabular}


Table A.10. Table 1A. (continued).

\begin{tabular}{|c|c|c|c|c|c|c|c|c|c|c|c|c|}
\hline 38570 & 38572 & 38588 & 38592 & 38594 & 38596 & 38598 & 38600 & 38602 & 38604 & 38612 & 38616 & 38618 \\
\hline 38620 & 38632 & 38634 & 38636 & 38652 & 38784 & 38786 & 38792 & 38794 & 38824 & 38826 & 38848 & 38850 \\
\hline 38856 & 38858 & 38888 & 38890 & 39040 & 39042 & 39046 & 39048 & 39050 & 39056 & 39058 & 39060 & 39064 \\
\hline 39066 & 39068 & 39072 & 39074 & 39076 & 39078 & 39080 & 39082 & 39084 & 39088 & 39092 & 39096 & 39100 \\
\hline 39104 & 39106 & 39108 & 39110 & 39112 & 39114 & 39116 & 39120 & 39122 & 39124 & 39128 & 39130 & 39132 \\
\hline 39136 & 39138 & 39140 & 39142 & 39144 & 39146 & 39148 & 39152 & 39156 & 39160 & 39164 & 39328 & 39330 \\
\hline 39332 & 39336 & 39338 & 39340 & 39360 & 39362 & 39364 & 39368 & 39370 & 39372 & 39392 & 39394 & 39396 \\
\hline 39400 & 39402 & 39584 & 39586 & 39588 & 39590 & 39592 & 39594 & 39596 & 39600 & 39604 & 39608 & 39612 \\
\hline 39616 & 39618 & 39622 & 39624 & 39626 & 39632 & 39636 & 39640 & 39644 & 39648 & 39650 & 39652 & 39656 \\
\hline 39658 & 39660 & 39664 & 39668 & 39672 & 39676 & 39840 & 39842 & 39844 & 39848 & 39850 & 39904 & 39906 \\
\hline 39912 & 39914 & 40096 & 40098 & 40104 & 40106 & 40112 & 40116 & 40120 & 40124 & 40160 & 40162 & 40164 \\
\hline 40168 & 40170 & 40172 & 40176 & 40180 & 40184 & 40188 & 40416 & 40418 & 40424 & 40426 & 40672 & 40674 \\
\hline 40680 & 40682 & 40688 & 40692 & 40696 & 40700 & 43008 & 43010 & 43012 & 43016 & 43018 & 43020 & 43028 \\
\hline 43032 & 43036 & 43048 & 43050 & 43052 & 43068 & 43072 & 43074 & 43076 & 43080 & 43082 & 43084 & 43092 \\
\hline 43096 & 43100 & 43112 & 43114 & 43116 & 43132 & 43136 & 43138 & 43140 & 43144 & 43146 & 43148 & 43156 \\
\hline 43160 & 43164 & 43176 & 43178 & 43180 & 43196 & 43200 & 43202 & 43204 & 43208 & 43210 & 43212 & 43220 \\
\hline 43224 & 43228 & 43240 & 43242 & 43244 & 43260 & 43266 & 43272 & 43274 & 43304 & 43306 & 43330 & 43336 \\
\hline 43338 & 43368 & 43370 & 43392 & 43394 & 43400 & 43402 & 43432 & 43434 & 43456 & 43458 & 43464 & 43466 \\
\hline 43496 & 43520 & 43522 & 43524 & 43528 & 43530 & 43532 & 43540 & 43544 & 43548 & 43560 & 43562 & 43564 \\
\hline 43580 & 43584 & 43586 & 43588 & 43592 & 43594 & 43596 & 43604 & 43608 & 43612 & 43624 & 43628 & 43644 \\
\hline 43648 & 43650 & 43652 & 43656 & 43658 & 43660 & 43668 & 43672 & 43676 & 43688 & 43690 & 43692 & 43708 \\
\hline 43712 & 43714 & 43716 & 43720 & 43722 & 43724 & 43732 & 43736 & 43740 & 43752 & 43756 & 43772 & 43778 \\
\hline 43784 & 43786 & 43816 & 43818 & 43842 & 43848 & 43850 & 43880 & 43904 & 43906 & 43912 & 43914 & 43944 \\
\hline 43968 & 43970 & 43976 & 43978 & 44008 & 44040 & 44042 & 44056 & 44064 & 44066 & 44068 & 44072 & 44084 \\
\hline 44088 & 44104 & 44106 & 44120 & 44128 & 44130 & 44132 & 44136 & 44148 & 44152 & 44160 & 44162 & 44164 \\
\hline 44168 & 44170 & 44176 & 44180 & 44184 & 44192 & 44194 & 44196 & 44200 & 44208 & 44212 & 44216 & 44224 \\
\hline 44226 & 44228 & 44232 & 44234 & 44240 & 44244 & 44248 & 44256 & 44258 & 44260 & 44264 & 44272 & 44276 \\
\hline 44280 & 44298 & 44322 & 44328 & 44362 & 44386 & 44392 & 44416 & 44418 & 44424 & 44426 & 44448 & 44450 \\
\hline 44456 & 44480 & 44488 & 44512 & 44552 & 44554 & 44568 & 44576 & 44578 & 44584 & 44616 & 44632 & 44640 \\
\hline 44648 & 44672 & 44674 & 44680 & 44682 & 44688 & 44696 & 44704 & 44706 & 44712 & 44736 & 44738 & 44744 \\
\hline 44752 & 44760 & 44768 & 44776 & 44810 & 44834 & 44840 & 44928 & 44936 & 44960 & 48168 & 48232 & 48256 \\
\hline 48258 & 48264 & 48296 & 48320 & 48322 & 48328 & 48360 & 48680 & 48744 & 48768 & 48770 & 48776 & 48808 \\
\hline 48832 & 48840 & 48872 & 59520 & 59528 & 59560 & 59584 & 59592 & 59624 & 60072 & 60096 & 60104 & 60576 \\
\hline 60640 & & & & & & & & & & & & \\
\hline
\end{tabular}

\section{References}

Chua, L. O., Guan, J., Sbitnev, V. I. \& Shin, J. [2007] "A nonlinear dynamics perspective of Wolfram's New Kind of Science. Part VII: Isles of Eden," Intern. Journal of Bifurcation and Chaos 17, 2839-3012. Chua, L. O., Sbitnev, V. I. \& Yoon, S. [2004] "A nonlinear dynamics perspective of Wolfram's New Kind 
of Science. Part III: Predicting the unpredictable." Intern. Journal of Bifurcation and Chaos 14, 3689-3820.

Chua, L. O., Sbitnev, V. I. \& Yoon, S. [2005] "A nonlinear dynamics perspective of Wolfram's New Kind of Science. Part IV: From Bernoulli shift to $1 / f$ spectrum," Intern. Journal of Bifurcation and Chaos 15, 1045-1183.

Guan, J., Shen, S., Tang, C. \& Chen, F. [2007] "Extending Chua's global equivalence theorem on Wolfram's New Kind of Science," Intern. Journal of Bifurcation and Chaos 17, 4245-4259.

Li, W. \& Packard, N. [1990] "The structure of the elementary cellular automata rule," Complex Systems 4, 281-297.

Packard, N. \& Wolfram, S. [1985] "Two-dimensional cellular automata," Journal of Statistical Physics 38, 901-946.

Walker, C. C. \& Aadryan, A. A. [1971] "Amount of computation preceding externally detectable steady sate behavior in a class of complex systems," J. Bio-Med. Comput. 2, 85-94.

Wolfram, S. [1984a] "Computation theory of cellular automata," Commun. Math.Phys. 96, 15-57.

Wolfram, S. [1984b] "Universality and complexity in cellular automata," Physica D 10, 1-35.

Wuensche, A. \& Lesser, M. [1992] The Global Dynamics of Cellular Automata, Santa Fe Institute Studies in the Sciences of Complexity (Addison-Wesley). 